\title{
The Emerging Thereat of Carbapenem Resistant Enterobacteriacae-CRE
}

\author{
Asim Ahmed Elnour ${ }^{1,2 *}$, Deema AL Hashmi ${ }^{3}$, Zakieh Mahmoud Alchikh ${ }^{3}$ and Akshaya Srikanth ${ }^{4}$ \\ ${ }^{1}$ Faculty-Pharmacy, Fatima College of Health Sciences, UAE \\ ${ }^{2}$ Adjunct Associate Professor, Monash University, Australia \\ ${ }^{3} 4$ th Year, Pharmacy program, FCHS, Abu Dhabi Campus, Al Ain UAE \\ ${ }^{4}$ Department of Clinical Pharmacy, College of Medicine and Health Sciences, Gondar University, Gondar, Ethiopia
}

\begin{abstract}
Received: April 28, 2016; Accepted: May 05, 2016; Published: May 13, 2016
*Corresponding author: Asim Ahmed Elnour, Faculty-Pharmacy, Fatima College of Health Sciences, UAE and Adjunct Associate Professor, Monash University, Australia, Tel: +971525437665, +96899791465; Email:asim.ahmed@hfchs.ac.ae; imad.nour@moh.gov.om
\end{abstract}

The common bugs of Enterobacteriacea are responsible for urinary tract infections, abdominal infections and hospitalacquired pneumonia. The resistance to Beta lactam in these dreadful bugs is most frequently due to the genetic expression of beta lactamase enzymes. The growing global concerns of Carbapenem resistant enterobacteriacea-CRE dictate a momentum to implement forcing-functions and prevent further wide spread of this global threat. The definition stated by the Center of Disease Control (CDC) define CRE as resistant to anycarbapenem antimicrobial (i.e., minimum inhibitory concentrations-MIC of $\geq 4 \mathrm{mcg} / \mathrm{ml}$ for Doripenem, Meropenem, or Imipenem OR $\geq 2 \mathrm{mcg} / \mathrm{ml}$ for Ertapenem), or documented to producecarbapenemase. CRE is a devastating threat that result in adverse consequences including high mortality rates and expedite transmission in facilities. Therefore, attempts to identify CRE their epidemiology, level of threat, recognition of infected patients and respective interventions are the most determent steps in climbing the uphill. The efforts to control the emerging CRE are multifactorial and more or less facility-specific. The interplay of global/regional/local public health, Healthcare facilities, nursing homes and the pharmaceutical industry is highly needed to coordinate the available strategic plans including contact precautions, antibiotic stewardship, communications and call for international collaboration with diverse stakeholders. The epidemiology of CRE encompasses the $\beta$-lactamases (such as AmpC and Extended Spectrum $\beta$-Lactamases-ESBL) and carbapenemases. The later has been implicated in Klebsiella pneumonia-KPcarbapenem resistance (KP CarbapenemaseKPC), New Delhi Metalo- $\beta$-Lactamase (NMB), Verona Integronencoded Metallo- $\beta$-Lactamase (VIM, Oxacillinase-48-type carbapenemases (OXA-48), and the Imipenemase (IMP) Metallo$\beta$-lactamase. Recognition of patients colonized or infected with
CRE represents the core for respective interventions to prevent and minimize the risk posed by transmission. Healthcare facilities need to have the ability to perform carbapenemase testing and CRE screening tests.

The forcing-functions of hand hygiene and contact precautions should be followed strictly. Limiting the use of devices is significant to prevent further device-associated source of CRE. The role of laboratory in following infection control protocols with immediate notifications is of paramount importance. The use of screening contacts personnel of CRE patients or active surveillance testing are deemed to strengthen the control of CRE. Lastly, the ongoing education of healthcare professional is inevitable to ensure a safety culture and further impact all other factors. There are new carbapenem introduced such as Panipenem/betamipron (not FDA approved) Biapenem and Tebipenem.Tebipenem is a novel broad-spectrum orallyadministered carbapenemprodrug form (pivalyl ester) developed in Japan as a replacement drug to combat resistance.

\section{References}

1. Chea N, Bulens SN, Kongphet-Tran T, Ruth Lynfield, Kristin M. Shaw, Paula Snippes Vagnone, et al. Improved phenotype-based definition for Identifying carbapenemase producers among CRE. Emerg Infect Dis. 2015;21(9):1611-1616. DOI: 10.3201/eid2109.150198.

2. Calfee D, SG Jenkins. Use of active surveillance cultures to detect asymptomatic colonization with carbapenem-resistant Klebsiellapneumoniaein intensive care unit patients. Infect. Control Hosp Epidemiol. 2008;29(10):966-968. doi: 10.1086/590661.

3. http://www.cdc.gov/HAI/pdfs/labSettings/Klebsiella_or_Ecoli.pdf

4. El-Gamal MI and $\mathrm{CH}$ Oh. Current status of carbapenem antibiotics. Curr Top Med Chem. 2010;10(18):1882-1897. 